\title{
A Noise-Tolerant Method for Measuring MTF from Found-Object Edges in a TEM
}

\author{
Paul E. Mooney
}

Gatan Inc., 5794 Las Positas Blvd., Pleasanton, CA 94566, USA

TEM camera MTF characterization usually involves analysis of found-object edges of uncontrolled orientation, straightness or cleanliness. In addition, the noise in TEM images is often higher than that readily attainable in a light-optical camera test due to shot and detector noise. The ISO12233 standard [1] defines a method for measurement of digital cameras using prefabricated resolution targets, high-contrast edges at a given slight tilt, imaged under bright conditions. Gatan has adapted the ISO standard for use in TEM camera characterization under a variety of less well controlled imaging conditions [2].

A sampled image of a tilted edge contains all the information needed to measure the MTF of the sampling image detector since pixels fall in general at all possible positions relative to the edge transition. The ISO12233 technique forms an oversampled image of a virtual edge by combining the samples from oblique edge crossings with effective sampling pitch of $p^{*} \tan (\theta)$ (where $p$ is the pixel pitch and $\theta$ is the tilt angle). For small angles this allows very high oversampling of the edge. This oversampled edge function can then be differentiated to form an effective image of a pseudo-line much narrower than the pixel, an FFT of that pseudo-line then yielding an nonaliased MTF of the combined image detector including the pixel sampling aperture. For TEM imager characterization, however, this technique is problematic. The first problem is that a very straight edge is required. Even the slightest bumpiness or curvature of the edge jumbles the pitch between successive samples as measured by the perpendicular distance to the edge. The second problem is that the differentiation of the oversampled image can be quite noisy unless the image is very well exposed. Both must be addressed for the images typically acquired in a TEM with 100-2000 primary electrons using in general curved test objects like a beam-stop edge.

This can be accomplished by looking at the series of near-perpendicular edge crossings instead of oblique edge crossings. Each crossing is differentiated, its centroid found, and the centroid location used to accumulate crossing profiles into $\mathrm{N}$ sub-pixel phase averages (figure 1a). The result is more noise-tolerant because the differentiation is done with a denominator of one pixel instead of the oversampling sub-pixel used in the ISO method. The $\mathrm{N}$ phase averages can then be unfolded into a single line-spread function analogous to that obtained in the ISO method (figure 1b), an FFT yielding a non-aliased MTF valid beyond Nyquist. The 1-pixel-wide pseudo-line source created by differentiation of the perpendicular edge crossings can be easily factored out of the oversampled MTF to first order by dividing by the sinc function and to higher accuracy by accounting for the tilt of the pixel aperture. Foreshortening of distances caused by the tilt is recalibrated in the oversampled LSF. Edge curvature up to a limit is handled by segmenting the edge into regions and rescaling the oversampled LSFs per segment before creating a full average and MTF. In order to correctly account for long-range effects such as light scatter in a 
lens/mirror system, no overall intensity calibration is done in the MTF calculation. Instead the input image is calibrated by taking the image pair: "edge inserted" and "edge retracted" and dividing to form a normalized edge image that includes long-range scatter effects (figure 2 ).

The algorithm has been tested with simulated-edge images created in 8-times oversampled coordinates, blurred by a known MTF model function in Fourier space and binned down to sensor pixel size. Figure 3 shows that the method applied to a "perfect" edge correctly measures MTF out to Nyquist for a range of tilt angles as being approximately equal to the sinc function. Noise immunity is demonstrated by adding shot noise to the simulated image. Figure 4 shows that it is advisable to use at least 1000 primary electrons to obtain accurate MTF results on a small (256-row) edge image. Adding more rows confers greater noise immunity. Comparisons of amorphous carbon Thon ring signal-to-noise ratios compensated by DQE using this MTF method on the same microscope with two different magnifications [ref. 2 - figure 14] and also on two different detectors [ref. 3 - figure 1d] have substantiated the accuracy of this MTF measurement technique in terms of specimen image quality.

\section{References}

[1] ISO 12233:2000(E), International Standards Organization (2000).

[2] Mooney, P., Meth. in Cell Biol., Vol. 79, Academic Press, San Diego, CA, 2007, 661-719.

[3] Luecken, U. et al, Microsc. Microanal. 14(Suppl 2) (2008) 1322.

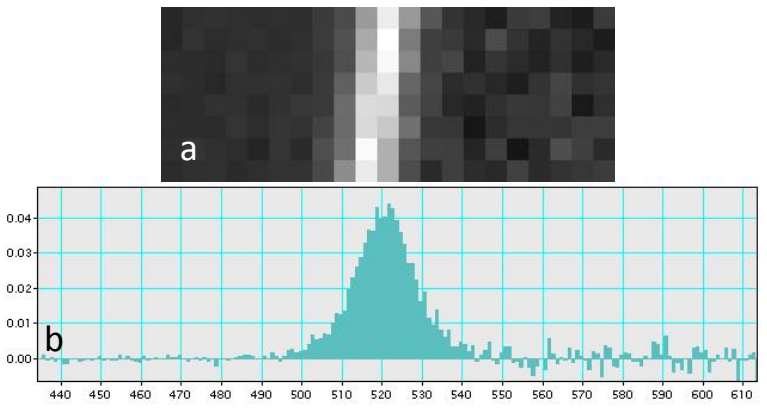

Figure 1. a. Selection from an image of 8 sub-pixel phase averages from a 64-row-high edge image. $b$. unfolded into oversampled LSF.

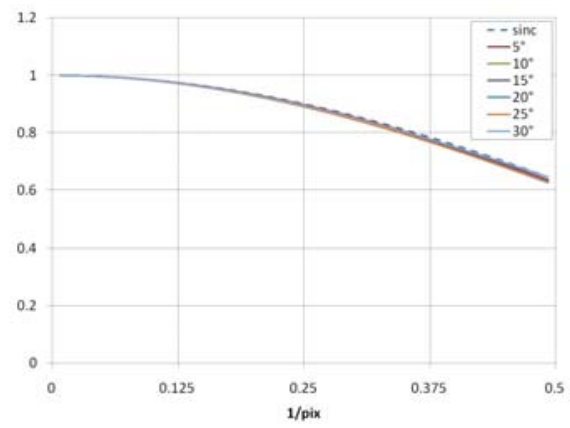

Figure 3. MTF algorithm with tilted aperture compensation applied to tilted-edge model images compared to the sinc function.

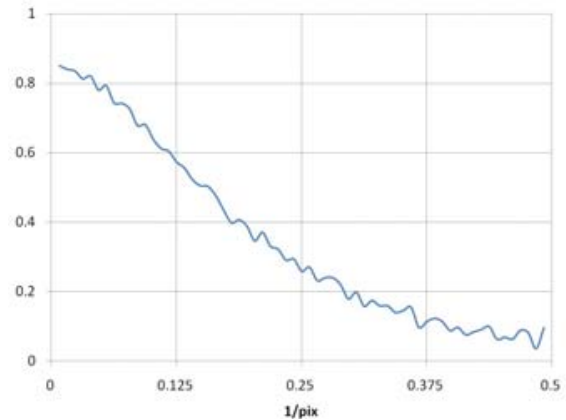

Figure 2. MTF of a mirror-lens-coupled scintillator showing correct measurement of light scatter at $\mathrm{s}=0$.

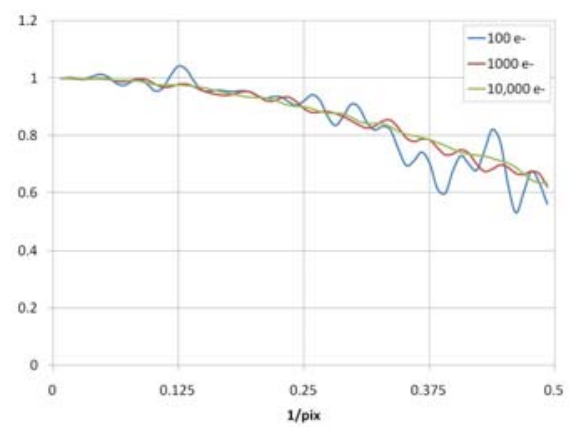

Figure 4. MTF of a $256 \times 2565^{\circ}$-tilted edge imaged with Poisson noise of 100, 1000 and 10,000 electrons added. 